\title{
Evaluation de l'impact de l'utilisation du diammonium phosphate sur la disponibilité du zinc dans un sol rizicole au Mali
}

\author{
Adama DIALLO $^{1 *}$, Boubacar TRAORE ${ }^{1}$, Souleymane DAMBE ${ }^{1}$, Sanata TRAORE $^{1}$, \\ Antoine Padou DIARRA ${ }^{1}$, Hamidou KONARE ${ }^{2}$, Brahima B. TRAORE ${ }^{1}$, \\ Mama PLEA ${ }^{1}$ et Lars ERICKSON ${ }^{3}$
}
${ }^{1}$ Laboratoire de Physico-chimie des matériaux de la Faculté des Sciences et Techniques de l'Université des Sciences, des Techniques et des Technologies Bamako, BPE 3206 Bamako, Mali.
${ }^{2}$ Laboratoire Sol-Eau - Plantes du Centre Régional de Recherche Agronomique de Sotuba de l'Institut d'Economie Rurale Bamako, BP 262 Bamako, Mali.
${ }^{3}$ Laboratoire Arrhénius Département de Chimie des matériaux et Environnement, Université de Stockholm, Suède.
*Auteur correspondant; Cel. (+223) 76101715 ; E-mail : karadam00@yahoo.fr

\section{RESUME}

L'utilisation des fertilisants chimiques dans l'agriculture s'est fortement accentuée ce dernier temps au Mali. En effet l'apport régulier du diammonium phosphate (DAP) peut influencer la mobilité du zinc, car les phosphates fixent cet élément. L'étude a pour but d'évaluer l'impact de l'utilisation de ce fertilisant phosphaté sur la disponibilité du zinc. Ainsi pour la caractérisation, l'échantillon est analysé par plusieurs méthodes physico-chimiques: la diffraction des rayons $\mathrm{X}$ et la microscopie électronique à balayage. Des essais d'absorption ont été réalisés avec cinq pots numérotés $\left(\mathrm{P}_{0}, \mathrm{P}_{1}, \mathrm{P}_{2}, \mathrm{P}_{3}, \mathrm{P}_{4}\right)$ contenant chacun $1 \mathrm{~kg}$ d'échantillon auxquels a été ajouté respectivement $0 ; 2 ; 4 ; 8$ et $10 \mathrm{~g}$ du fertilisant. D'autres essais ont été conduits avec $3 \mathrm{~g}$ d'échantillons prélevés dans le pot $\mathrm{P}_{4}$, auxquels ont été appliqué respectivement 150 ppm, 300 ppm, 450 ppm et 600 ppm de zinc. Il ressort de ce travail que la quantité de $\mathrm{Zn}$ retenu est proportionnelle à celle du DAP et que la limite (rétention en 21 jours) $=499,9993 \mathrm{ppm}>$ limite (rétention en 15 jours) $=499,9991 \mathrm{ppm}>$ limite (rétention en 7 jours $)=499,9990 \mathrm{ppm}>$ limite (rétention en 1 jour) $=499,9980 \mathrm{ppm}$. Cette étude a montré que le diammonium phosphate retient le zinc et influence donc sa biodisponibilité.

(c) 2020 International Formulae Group. All rights reserved.

Mots clés : Sol rizicole, Pana au Mali, rétention du zinc, impact de l'utilisation, Diammonium phosphate.

\section{Estimate the impact of phosphate diammonium usage on the availability of zinc in a rice producing soil in Mali}

\begin{abstract}
The use of chemical fertilizers in agriculture has highly increased this last time in Mali. In fact the regular contribution of DAP can influence the mobility of zinc, because the phosphate fix this element. The object of this study is to estimate the impact of this phosphate fertilizer on the availability of zinc. So for the characterization, the sample is analysed through several physico-chemical methods: the $\mathrm{X}$ rays diffraction and
\end{abstract}


the sweeping electronic microscopy. Some absorbing tests have been carried out with five numbered pots $\left(\mathrm{P}_{0}\right.$, $\mathrm{P}_{1}, \mathrm{P}_{2}, \mathrm{P}_{3}, \mathrm{P}_{4}$ ) containing each $1 \mathrm{~kg}$ of sample to which has been added respectively $0 ; 2 ; 4 ; 8$ and $10 \mathrm{~g}$ of the fertilizer. Other tests have conducted with $3 \mathrm{~g}$ samples removed from the pot $\mathrm{P}_{4}$, to which has been applied respectively $150 \mathrm{ppm}, 300 \mathrm{ppm}, 450 \mathrm{ppm}$ and $600 \mathrm{ppm}$ of zinc. As a result of the work the quantity of zinc retained is proportional to the one of DAP and the limit (retention in 21 days) $=499.9993 \mathrm{ppm}>$ limit (retention in 15 days $)=499.9991 \mathrm{ppm}>$ limit $($ retention in 7 days $)=499.9990 \mathrm{ppm}>$ limit (retention in 1 day $)=499.9980$ ppm. This study has shown that the phosphate diammonium retains the zinc and influences in this case its bioavailability.

(C) 2020 International Formulae Group. All rights reserved.

Keywords: Rice producing soil, Pana in Mali, zinc retention, the impact of the usage, phosphate diammonium.

\section{INTRODUCTION}

Le zinc est un oligo-élément essentiel pour la vie des plantes et des animaux notamment à cause de ses effets bénéfiques pour le système immunitaire chez les êtres vivants. Les sols en contiennent 10 à $300 \mathrm{mg}$ par kg (Pichard, 2005). Dans le sol, cet élément a une grande affinité vis-à-vis du phosphore. Le phosphore, spécifiquement l'orthophosphate est un captieux nutriment qui peut être utilisé par les microorganismes et les plantes (Chunsheng et al., 2013 ; Frossard et al., 2004). Le diammonium phosphate (DAP) est un fertilisant chimique beaucoup plus utilisé dans la culture du riz et d'autres céréales au Mali. Son utilisation augmente aussi le rendement de la production du niébé (Mulambuila Nkongolo et al., 2015 ; Michel et al., 2017). Les effets du phosphate sur la mobilité du zinc ont fait l'objet d'étude précise (Pardo, 2008). Son influence dans les dynamiques de ce métal dans les sols est très intéressante (Gertrud, 2005). Les produits alimentaires dérivés de l'agriculture sont déficients en zinc et en phosphore dans de nombreuses zones du monde (Pérez-Novo et al., 2011 ; Pardo, 2008). Cette déficience en zinc est due en partie à l'intensification des pratiques agricoles et la pauvreté de la matrice sol en cet élément (Maliki et Ifijem, 2020). Les interactions phosphore-zinc ont été largement étudiées (Vega et al., 2007). Plusieurs mécanismes ont été proposés pour expliquer les interactions du zinc et du phosphore (Pardo, 2008). L'augmentation de la quantité de phosphore dans un milieu de culture par addition du DAP peut induire une déficience en zinc dans les sols et les tissus des végétaux. Tout de même, on dispose de peu d'informations sur l'effet du phosphore sur la biodisponibilité du zinc et de la concentration du zinc dans les tissus végétaux (Pahlavan-Rad et Pessarakli, 2009). Les éléments traces métalliques (ETMs) sont fixés dans le sol sous un nombre restreint de formes. Les quatre espèces zincifères sont le phosphate de type $\left[\mathrm{Zn}_{3}\left(\mathrm{PO}_{4}\right)_{2} .4 \mathrm{H}_{2} \mathrm{O}\right]$; l'oxyde de fer mal cristallisé ; le phyllosilicate trioctaédriaque de type $\mathrm{Si}_{4}\left(\mathrm{Mg}_{3-\mathrm{x}} \quad \mathrm{Zn}_{\mathrm{x}}\right) \quad \mathrm{O}_{10}(\mathrm{OH})_{2} \mathrm{nH}_{2} \mathrm{O}$ et le willémite $\mathrm{Zn}_{2} \mathrm{SiO}_{4}$. Bien que les connaissances acquises soient de nature fondamentale, c'est un domaine dans lequel le transfert de technologie est rapide. Dans le sol la matière organique retient très peu le zinc (Panfili, 2004). Cependant, peu de sols présentent du zinc sous forme soluble et la mobilité du zinc est donc limitée par un faible taux de dissolution. Il est rapidement converti dans ses formes chimiques les moins actives (complexes organiques et surtout les précipités minéraux (Adhikari et al., 2007). Pour corriger la déficience du zinc dans les sols il faut appliquer 5 à $20 \mathrm{~kg}$ de zinc à l'hectare. Le sulfate de zinc est le plus souvent utilisé. Dans les phases liquide et solide des sols, il existe des équilibres chimiques entre le zinc et certains composants. A cet effet, les formes sous lesquelles le zinc est couramment rencontré sont : $\mathrm{Zn}(\mathrm{OH})_{2}, \mathrm{ZnL}$, $Z n A_{2}$, etc où $\mathrm{L}$ est un ligand et $\mathrm{A}$ un anion (Alloway, 2008). 
L'application du DAP est annuelle et régulière dans les rizières de PANA au Mali. La norme de ce fertilisant appliquée est $100 \mathrm{~kg}$ par hectare soit 0,03 g de DAP par kg de terre (Theriant et al., 2015). Le constat est qu'on assiste de plus en plus à une saturation des sols en phosphate dû au non-respect des normes et à la régularité de l'application de ce fertilisant qui pourrait être dommageable pour la mobilité du zinc qui joue un rôle précieux dans la vie des plantes et des animaux. Face à cette situation le laboratoire de Physico-Chimie des Matériaux de la Faculté des Sciences et Techniques de Bamako en collaboration avec le Centre National de Recherche Agronomique de Sotuba au Mali a mené un travail d'expérimentation portant sur l'évaluation de l'impact de l'utilisation abusive du DAP sur la disponibilité du zinc dans les sols agricoles. L'étude a eu pour objectif de contribuer à la préservation de la qualité des sols rizicoles et des cultures vivrières au Mali pour le bien-être de la population.

\section{MATERIEL ET METHODES}

\section{Caractéristiques générales de la zone d'étude}

L'échantillonnage a eu lieu à PANA dans la commune rurale de MOUGNA au Mali, cercle de Djénné dans la région de Mopti. Sa situation géographique est indiquée sur la carte ci-après (Figure 1).

C'est une zone semi-aride située dans le delta central du fleuve Niger. Elle est emprisonnée dans l'inter fleuve Bani-Niger. C'est aussi une zone de dépôt sédimentaire alluviale des deux cours d'eau. Elle fait une superficie de $35000 \mathrm{~km}^{2}$. Le climat est de type sahélien caractérisé par une saison pluvieuse de juillet à septembre et une saison sèche de novembre à mai. La hauteur des précipitations est comprise entre $400 \mathrm{~mm}$ et $600 \mathrm{~mm}$ (Bationo et al., 2006).

\section{Matériel}

Les prélèvements sont effectués à l'horizon $(0-20 \mathrm{~cm})$ selon la procédure standard du laboratoire sol-eau-plante du Centre National de Recherche Agronomique (CNRA) de Sotuba de l'Institut d'Economie Rurale (IER) Bamako (Handbook, 1992) dans un chanp situé dans les rizières du village de PANA qui sont des plaines aux bords du fleuve Bani. Les coordonnées géographiques du champ sont N 1351'59'" et W00444'30'.

Les échantillons ont été transportés au laboratoire dans des sacs en polyéthylène et ensuite séchés à l'air. Dans cette étude nous avons procédé par une approche pédagogique simple. Nous avons choisi un échantillon de sol argileux avec $43 \%$ fraction inférieure à $2 \mu \mathrm{m}$ obtenu par mélange des prélèvements de sol en dix endroits du champ et reparti dans cinq pots identiques, auxquels est ajouté du DAP pour incubation durant de 45 jours. Le choix du taux d'argile $43 \%$ n'est pas fortuit. Il est dû au fait que la culture du riz en général se fait sur les sols argileux c'est-à-dire des sols dont la fraction fine inférieure à $2 \mu \mathrm{m}$ dépasse $35 \%$. Les quantités du DAP appliqué aux échantillons varient graduellement de 0, 2, 4, 8 et $10 \mathrm{~g}$, et cela dans le but d'évaluer la capacité de rétention du zinc par le DAP. Ces valeurs 2, 4,8 et $10 \mathrm{~g}$ valent respectivement $40 ; 80$; 160 et 200 fois celle de la norme de DAP appliqué au Mali. Le contenu de chaque pot recevait de l'eau chaque deux jours pendant toute la durée de l'incubation et cela pour la dissolution du fertilisant dans le sol. Dans le système de classification FAO, cet échantillon composite de PANA est un Cambisol vertique kaolinitique. Dans les sols la matière organique et l'argile sont deux agents principaux de fixation des cations minéraux et métallique. La matière organique (M.O.) a une capacité d'échange cationique élevée (200 à 300 méq/100 g) mais, ces types de sol ont peu de M.O. ( $\leq 2 \%)$. C'est l'argile par son abondance (dans ce cas 43\%) et surtout sa nature minéralogique qui va déterminer la capacité d'échange. Or l'essentiel du cortège minéral est constitué de kaolinite dont la capacité spécifique est faible (inférieure à 10 méq/100 g) (Anonyme, 2001). Ces caractéristiques ci- 
dessus décrits permettent d'apprécier le rôle du DAP dans l'échantillon incubé car la M.O. et l'argile interviennent très peu dans ce processus d'échange avec les ions métalliques dans ces genres sols.

\section{Caractérisation physico-chimique des sols étudiés}

L'échantillon de sol a été caractérisé par la détermination de la granulométrie (la méthode de la Pipette Robinson), du pH, de la matière organique (M.O.), de la capacité d'échange cationique (CEC), de la teneur de l'oligo-élément $\mathrm{Zn}$ et par l'analyse minéralogique de la fraction fine $<2 \mu \mathrm{m}$.

Le $\mathrm{pH}$ du sol a été déterminé avec un $\mathrm{pH}$-mètre dans une solution de sol où le rapport solution/sol est de $2,5 \mathrm{~mL} / 1 \mathrm{~g}$ (eau/sol). La texture $\mathrm{du}$ sol a été déterminée après destruction de la M.O. par l'eau oxygénée par la méthode de la Pipette Robinson (AFNOR., 2003). Le pourcentage de M.O. est déterminé par la méthode Anne modifiée consistant à oxyder le carbone du sol par un mélange de $\mathrm{K}_{2} \mathrm{CrO}_{7}+\mathrm{H}_{2} \mathrm{SO}_{4}$. Le phosphore assimilable a été estimé par la méthode BRAY II. La Capacité d'Echange Cationique (CEC) est déterminée par lessivage du sol par une solution de $\mathrm{CH}_{3} \mathrm{COONH}_{4}$ (Handbook, 1992): C'est la méthode du complexe absorbant. La quantité de $\mathrm{NH}_{4}{ }^{+}$retenue par le sol après lavage de l'excès d'acétate d'ammonium est une estimation de la CEC. Le zinc est déterminé par l'extraction au Diéthylène Triamine Penta Acétique (DTPA). La méthode consiste à mettre en contact dans un rapport $1 / 2(\mathrm{~m} / \mathrm{v})$ le sol et une solution de Triéthanol Amine (TEA) 0,1 $\mathrm{M}+0,01 \mathrm{M}$ de $\mathrm{CaCl}_{2}+0,005 \mathrm{M}$ de DTPA. Le pouvoir chélatant du DTPA permet une mise en solution partielle de cet élément (Van Der Pol et al., 1998). Cet élément est ensuite dosé dans l'extrait filtré par l'absorption atomique (Pekin Elmer Analyst 200). Le zinc total est déterminé après dissolution totale par l'eau régale qui est un mélange de $\mathrm{HCl} 37 \% / \mathrm{HNO}_{3} 65 \%$ dans un rapport volumique de 3/1. Pour l'analyse minéralogique, le diffractogramme de poudre a été réalisé à l'aide d'un diffractomètre Xpert Pro Powder Diffractometer From Panalytical, utilisant la radiation $\mathrm{CuK} \alpha$ de longueur d'onde $\lambda=1.54178 \AA$ en mode réflexion et fonctionnant sous $45 \mathrm{kV}, 40 \mathrm{~mA}$, l'image au microscope électronique à balayage est obtenue à l'aide du microscope Model TM3000 TABLETOP MICROSCOPE.

\section{Essais d'absorption du DAP par le sol}

Des essais d'absorption ont été réalisés dans cinq pots numérotés de 0 à $4\left(\mathrm{P}_{0} ; \mathrm{P}_{1} ; \mathrm{P}_{2}\right.$; $\left.\mathrm{P}_{3} ; \mathrm{P}_{4}\right)$ contenant chacun un kilogramme d'échantillon de sol de PANA, auxquels a été ajouté respectivement $0 \mathrm{~g} ; 2 \mathrm{~g} ; 4 \mathrm{~g} ; 8 \mathrm{~g}$ et 10 $\mathrm{g}$ de DAP. Cette étude a nécessité deux expériences :

$1^{\text {ère }}$ expérience : elle a consisté à mettre dans chaque pot, $3 \mathrm{~g}$ de mélange (échantillon+ DAP) prélevés et $50 \mathrm{ml}$ d'une solution de sulfate de zinc de concentration $500 \mathrm{ppm}$ pendant $24 \mathrm{~h}$ (1 jour) à $25^{\circ} \mathrm{C}$. La solution ainsi récupérée est ensuite passée à la centrifugation et la concentration du zinc dans le surnageât est déterminée par absorption atomique. La quantité de zinc retenu par l'échantillon de sol est calculée par différence. La même expérience c'est-à-dire mélange (échantillon + DAP) avec la solution de sulfate de zinc 500 ppm pendant 7 jours, 15 jours et 21 jours à 25 ${ }^{\circ} \mathrm{C}$ a été aussi réalisée afin d'étudier la cinétique de la rétention.

$2^{\text {ème }}$ expérience: elle a été réalisée avec le sol du pot $\mathrm{P}_{4}$ qui avait été incubé avec $10 \mathrm{~g}$ de DAP. A $3 \mathrm{~g}$ de ce sol, il a été aussi appliqué $50 \mathrm{~mL}$ de solution de $\mathrm{Zn}^{2+}$ de concentration $150 \mathrm{ppm}$. Nous avons répété la même expérience avec des solutions de sulfate de zinc de concentrations respectives $300 \mathrm{ppm} ; 450 \mathrm{ppm}$ et $600 \mathrm{ppm}$. Les essais sont conservés pendant 24 heures à $25^{\circ} \mathrm{C}$. Les surnageâts sont ensuite passés à la centrifugation et la concentration du zinc restant y est déterminée par absorption atomique. 

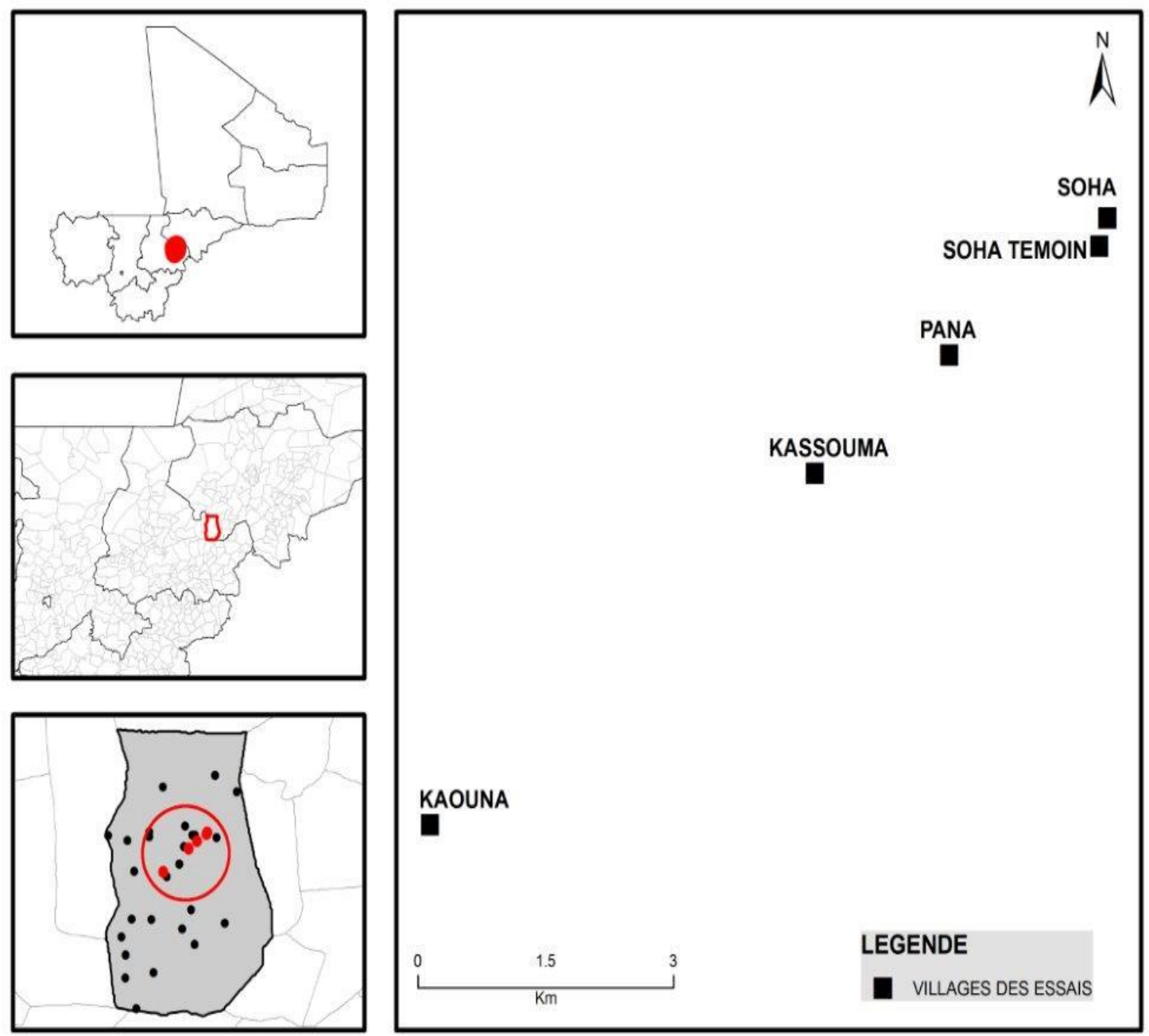

Figure 1 : Carte de la zone d'échantillonnage.

\section{RESULTATS}

\section{Caractéristiques générales du sol étudié}

Les valeurs moyennes obtenues sur les paramètres sont présentées dans le Tableau 1 ci-après.

Le $\mathrm{pH}$ exprime l'activité ou la concentration en ion $\mathrm{H}^{+}$et mesure l'acidité ou l'alcalinité d'un sol lue sur échelle allant de 0 à 14. Le pH constitue un facteur dont le rôle est crucial pour la mobilité des ions métalliques. Le $\mathrm{pH}$ mesuré dans l'échantillon est 4,9. Il présente un caractère fortement acide.

La matière organique du sol est composée d'organismes vivants, des résidus de végétaux et d'animaux et de produits en décomposition. Elle comprend tous les matériaux renfermant du carbone dans le sol. Elle joue un rôle important dans la fertilité du sol, même si elle est présente en quantité relativement faible (1 à 10\%) (Munroe, 2018). Elle stocke et fournit les éléments nutritifs, améliore la structure et l'infiltration de l'eau, favorise l'activité biologique et maintient le $\mathrm{pH}$ du sol. La matière organique de ce sol provient des dépôts alluvionnaires et des tiges sèches de riz après la récolte. La teneur en matière organique $(2,08 \%)$ est moyennement organique. La granulométrie indique $43 \%$ de 
fraction inférieure à $2 \mu \mathrm{m}$. Dans la classification texturale c'est un sol argileux. La capacité d'échange cationique 7,84 méq/100g est faible ce qui montre que le minéral argileux est la kaolinite. La teneur habituelle agricole en zinc de l'échantillon est 14,5 ppm comparé aux valeurs de Colinet (2003) montre qu'il n'est ni déficient ni toxique. L'image MEB (Figure 2)

\section{Rétention du Zn par les sols}

Les courbes de la Figure 4 sont relatives à la rétention du zinc par le DAP dans différents cas de figure. Elle correspond à la première expérience et montre que la quantité de zinc retenu augmente avec la quantité de DAP et avec le temps. Cela est matérialisé par la superposition des quatre courbes de rétention. Au-delà de 21 jours la rétention devient très faible. Dans le pot $\mathrm{P}_{0}$ sans DAP appliqué, nous constatons quand même une certaine rétention. Mais elle est faible par rapport à celle des autres pots. indique la structure de l'échantillon. Il apparait sur le cliché la forme hexagonale caractéristique de la kaolinite. Le diffractogramme aux rayons $X$ (Figure 3) réalisé sur ce même échantillon montre une faible quantité de kaolinite mal cristallisée et une forte quantité de quartz.

De $\mathrm{P}_{3}$ à $\mathrm{P}_{5}$ c'est-à-dire entre 4 et $10 \mathrm{~g}$ de DAP appliqué, les courbes de rétention sont sensiblement parallèles à l'axe des abscisses

Dans la $2^{\text {ème }}$ expérience, les résultats sont illustrés par la courbe de la Figure 5.

Elle indique la variation du zinc disponible en fonction du zinc appliqué. Nous constatons que la quantité de zinc non disponible croit avec la quantité de zinc appliqué au sol.

Tableau 1 : Valeurs des paramètres physico-chimiques de l'échantillon.

\begin{tabular}{ll}
\hline Les paramètres & Valeurs \\
\hline $\mathrm{pH}$ H2O & 4,9 \\
M.O. $(\%)$ & 2,08 \\
$\mathrm{CEC}($ méq/100g) & 7,84 \\
$\mathrm{Zn}_{\text {total }}(\mathrm{ppm})$ & 14,5 \\
$\mathrm{Zn}$ DTPA $(\mathrm{ppm})$ & 0,4 \\
Sable $(\%)$ & 24 \\
Limon $(\%)$ & 33 \\
Argile $(\%)$ & 43 \\
$\mathrm{P}$ assimilable (ppm) & 9,24 \\
Na échangeable (méq/100g) & 3,40 \\
$\mathrm{~K}$ échangeable (méq/100g) & 1,50 \\
Ca échangeable (méq/100g) & 0,42 \\
$\%$ saturation & $78,06 \%$ \\
\hline
\end{tabular}




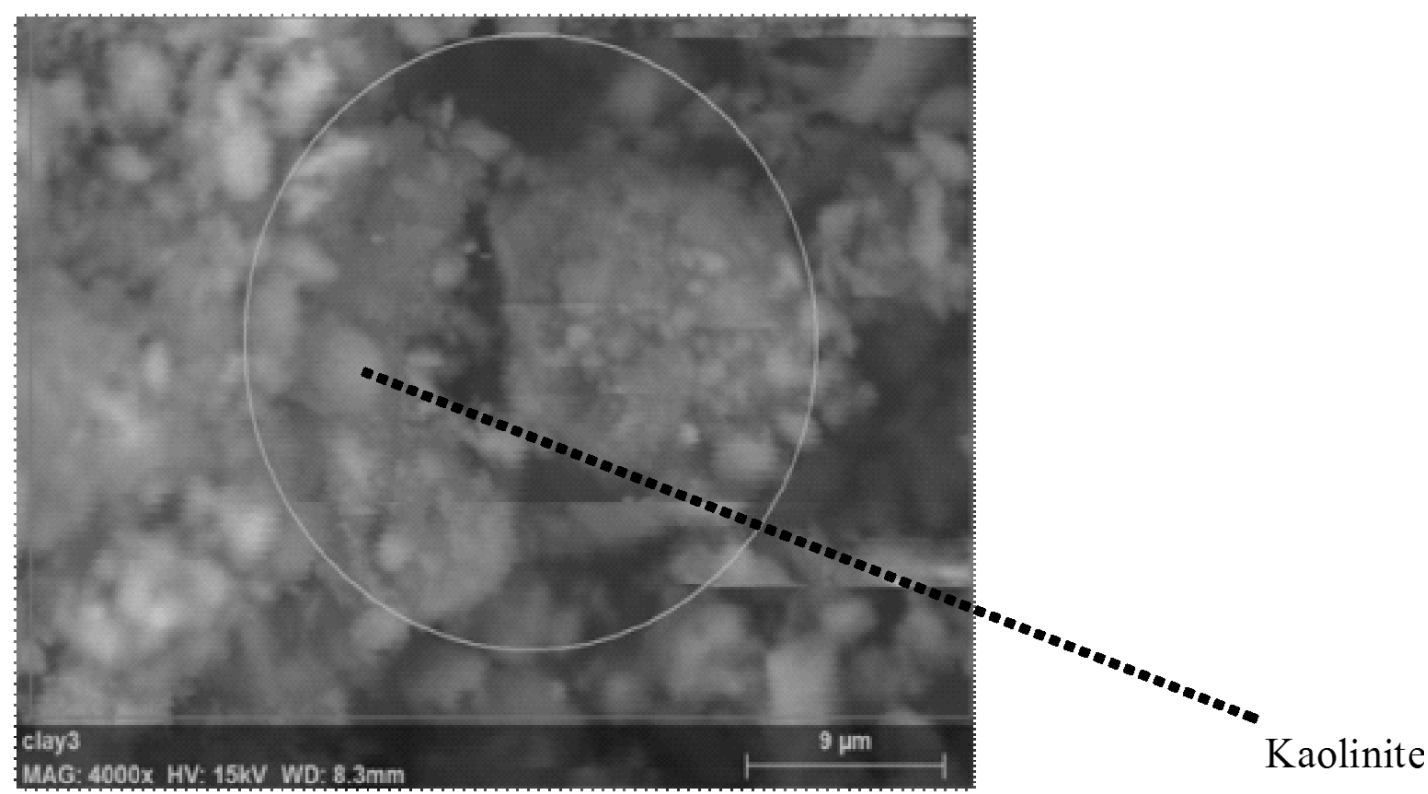

Figure 2 : Image MEB du sol de PANA.

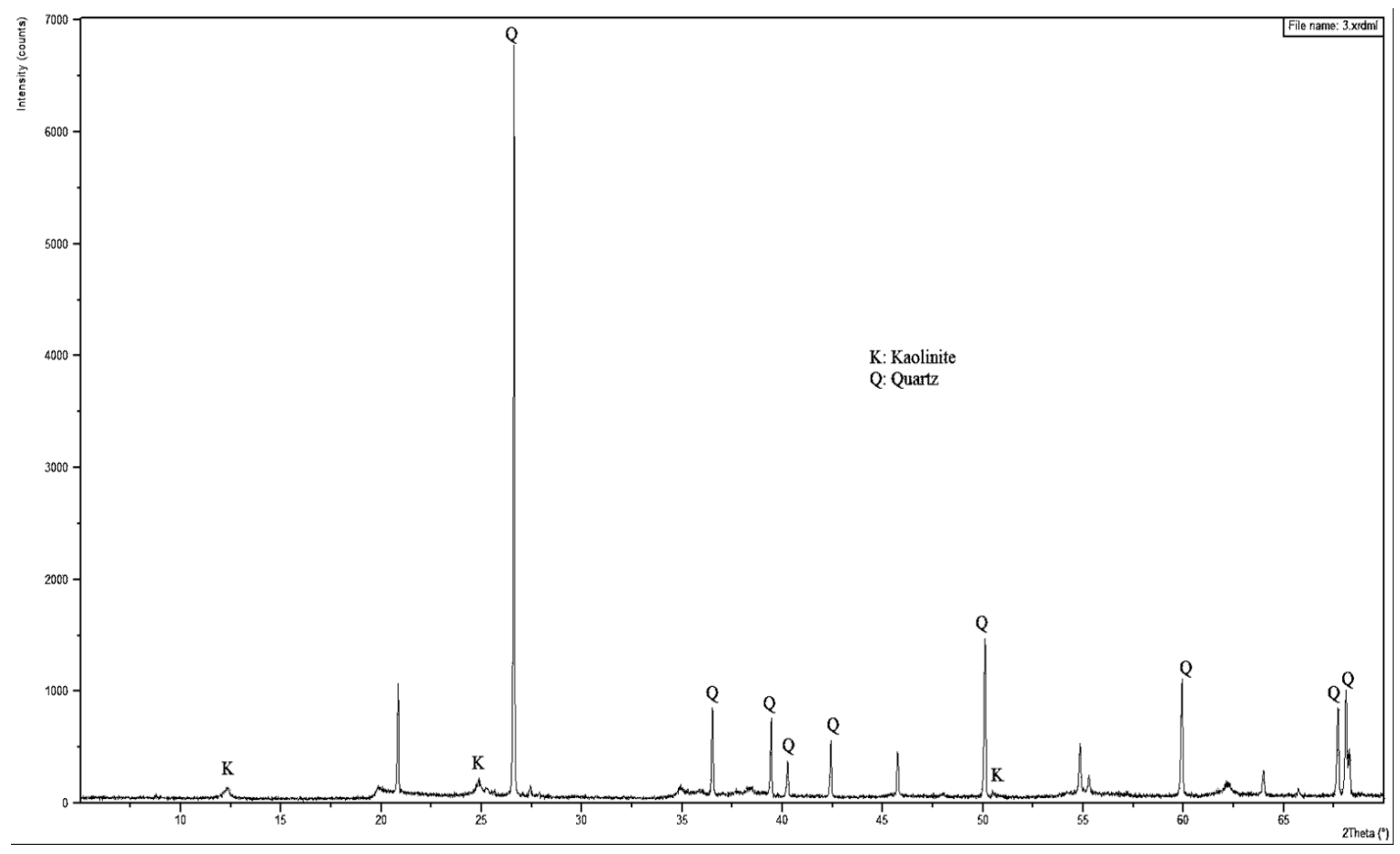

Figure 3 : Diffractogramme aux rayons X (DRX) du sol de PANA (angle 2 $\theta$ ). 


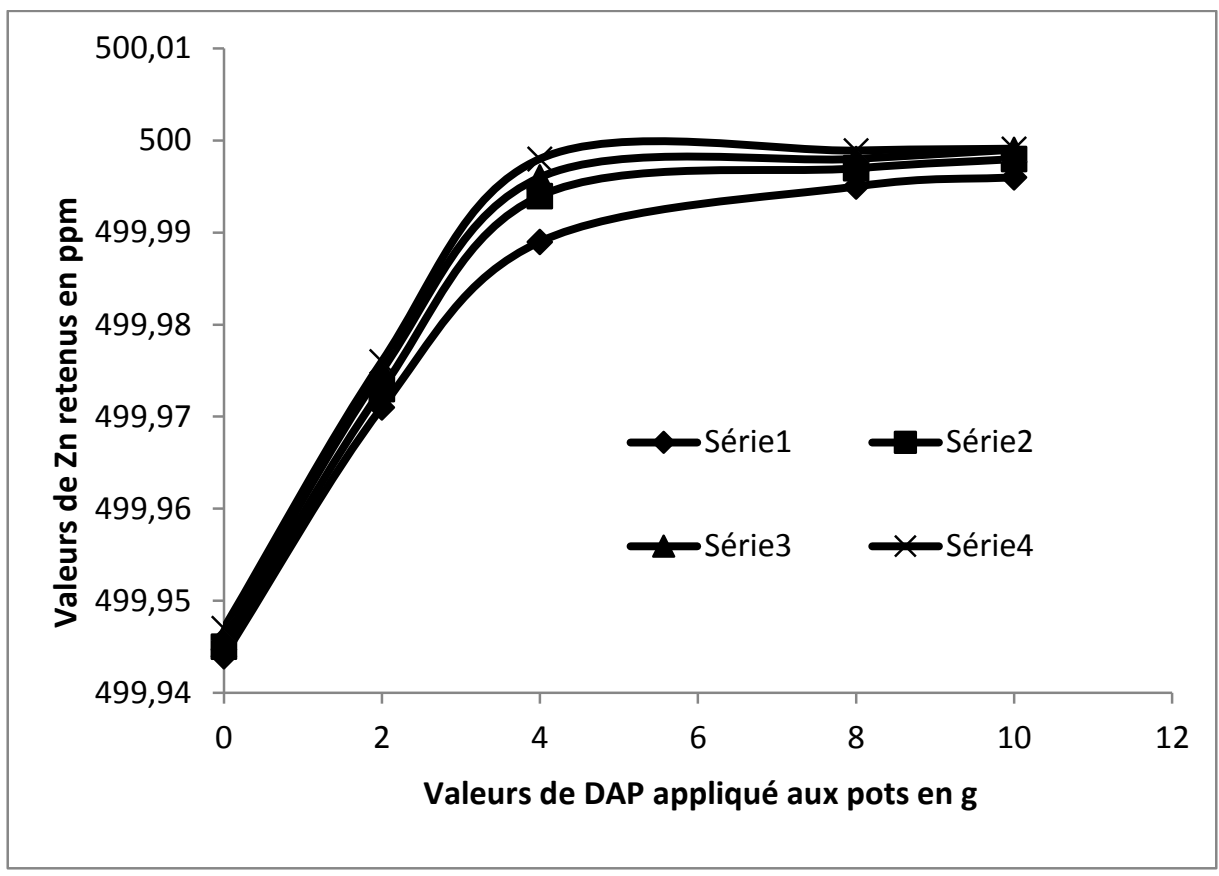

Figure 4 : Quantité de zinc retenu en fonction de la quantité de DAP appliquée aux pots 1jours (série4), 7jours (série3), 15jours (série2), 21 jours (série1) après la mise en contact avec la solution de sulfate de zinc.

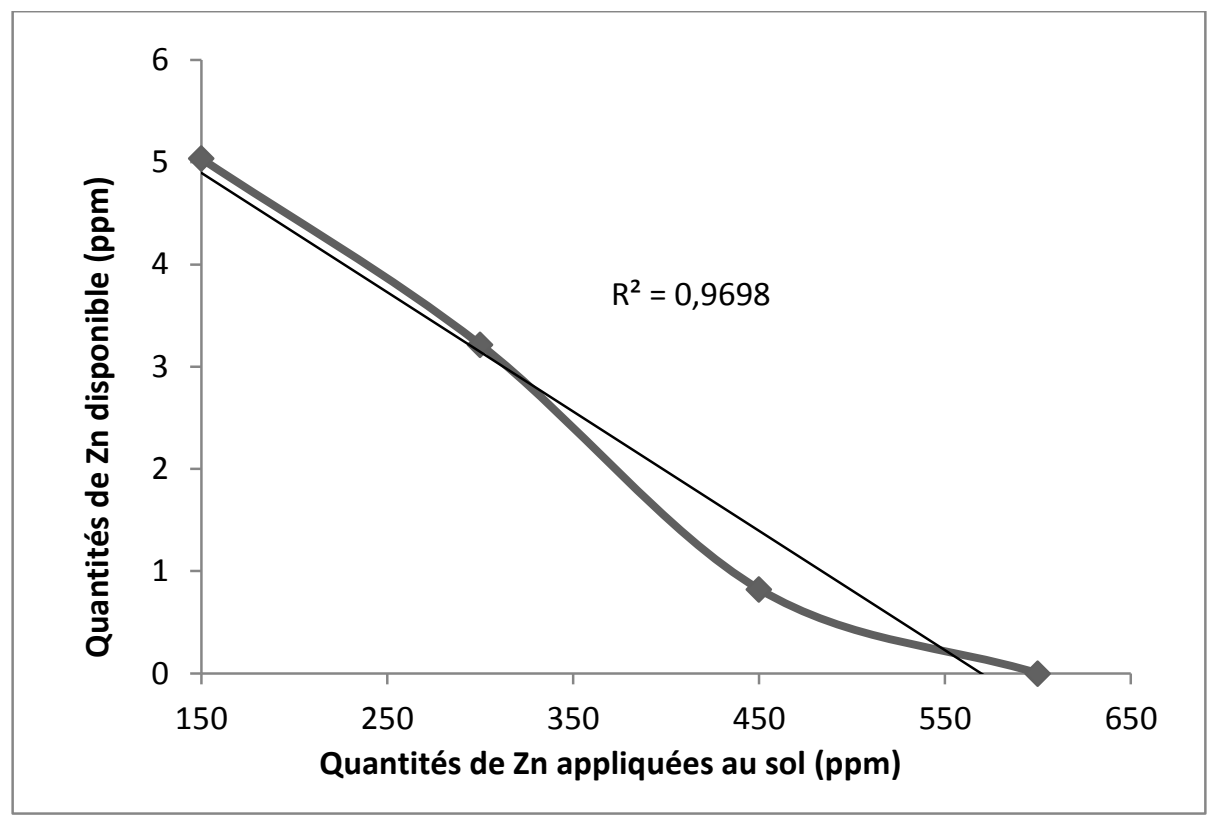

Figure 5 : Effet du zinc appliqué sur la quantité de zinc disponible. 


\section{DISCUSSION}

Le taux en matière organique moyennement élevé dépend du dépôt sédimentaire alluviale de crues saisonnières du fleuve Bani rive gauche entre les deux cours d'eau (Niger et Bani). La forte acidité est probablement due au mauvais drainage et la décomposition de la matière organique du sol sous l'effet conjugué de la forte chaleur et de l'humidité dans la zone.

Sur la figure 4, le pot $\mathrm{P}_{0}$ qui est le pot témoin sans DAP affiche une rétention de zinc. Mais la quantité retenue est inférieure à celles des pots contenant le DAP. Elle est probablement due à la charge négative de l'argile du sol et les autres composants chimiques tels que les oxydes, la matière organique etc. De $\mathrm{P}_{0}$ à $\mathrm{P}_{3}$ c'est-à-dire entre 0 et $4 \mathrm{~g}$ de DAP appliqué, les courbes sont pratiquement linéaires et ascendantes. Ce qui signifie que si les quantités de DAP appliqué ne sont pas suffisantes, le nombre d'ions de zinc retenu augmente avec le temps. Ce temps est relativement long car la limite de rétention n'est pas vite atteinte. Nous atteignons donc un palier pour une valeur suffisante de DAP (valeur supérieure à $4 \mathrm{~g}$ ). Les ions zinc de la solution de sulfate de zinc sont pratiquement tous retenus par le sol dopé de DAP en même temps. Alors la rétention cesse d'augmenter. La limite de rétention est atteinte. L'observation de la figure 4 montre aussi que les quatre courbes qui matérialisent la cinétique de rétention varient de façon similaire. La différence se situe au niveau de la limite de rétention du zinc : limite (rétention en 21 jours) $=499,9993 \mathrm{ppm}>$ limite (rétention en 15 jours) $=499,9991 \mathrm{ppm}>$ limite (rétention en 7 jours) $=499,9990 \mathrm{ppm}>$ limite (rétention en 1 jour) = 499,9980 ppm. La limite de rétention dépend du temps de contact de l'échantillon avec la solution de sulfate de zinc. Le facteur temps (cinétique) améliore le taux de rétention.

Il a été établi par certains auteurs (Anonyme, 2013) que suivant les valeurs du $\mathrm{pH}$, les ions phosphates $\left(\mathrm{H}_{2} \mathrm{PO}_{4}^{-}, \mathrm{HPO}_{4}^{2-}\right.$,
$\mathrm{PO}_{4}^{3-}$ ) peuvent piéger le zinc par un processus qui peut être schématisé entre ces ions phosphates et les cations zinc par :

Si le $\mathrm{pH}<6,8$, milieu acide l'anion $\mathrm{H}_{2} \mathrm{PO}_{4}^{-}$ domine dans la solution de sol

$$
2 \mathrm{H}_{2} \mathrm{PO}_{4}^{-}+\mathrm{Zn}^{2+} \leftrightharpoons \mathrm{Zn}\left(\mathrm{H}_{2} \mathrm{PO}_{4}\right)_{2}(\mathrm{I})
$$

Pour des valeurs comprises entre [6,8 - 9] l'anion $\mathrm{HPO}_{4}^{2-}$ domine

$$
\mathrm{Zn}^{2+}+\mathrm{HPO}_{4}^{2-} \leftrightharpoons \mathrm{ZnHPO}_{4} \text { (II) }
$$

Si le $\mathrm{pH}>9$ milieu basique c'est le $\mathrm{PO}_{4}^{3-}$ qui domine

$3 \mathrm{Zn}^{2+}+2 \mathrm{PO}_{4}^{3-} \leftrightharpoons \mathrm{Zn}_{3}\left(\mathrm{PO}_{4}\right)_{2}$ (III)

Dans cette étude le $\mathrm{pH}$ du sol est 4,9. Le $\mathrm{pH}$ étant fortement acide, nous pouvons dire que pratiquement tout le processus de rétention entre les ions zinc et les phosphates se limite à la seule réaction (I).

Ces deux facteurs c'est-à-dire la quantité et le temps permettent non seulement d'accroitre la probabilité de rencontre entre les différentes molécules de la réaction, mais aussi de favoriser les échanges d'ions avec les minéraux du sol et l'absorption des ions par la surface des matières argileuses de ce sol. Le phosphate de zinc est un composé très hydrophile, ce qui fait qu'il existe sous la forme hydratée $\left[\mathrm{Zn}_{3}\left(\mathrm{PO}_{4}\right)_{2} 2 \mathrm{H}_{2} \mathrm{O}\right]$ dans un milieu aqueux.

Nous remarquons sur la figure 5 que pour les faibles concentrations de zinc dans le sol en présence du DAP, la rétention du zinc est faible. La réaction entre les cations $\mathrm{Zn}^{2+}$ et les ions phosphates est comme toutes les autres réactions. Elle dépend de la probabilité de rencontre entre les molécules des corps réagissant (Frossard et al., 2004). Cette étude montre que le phosphate est l'un des responsables majeurs de la rétention du zinc dans les sols d'agriculture.

En général, le zinc est un oligo-élément qui est déficient dans les sols. L'utilisation de quantités excessives de fertilisant chimique comme le DAP peut aggraver cette déficience et engendrer plusieurs conséquences telles que : la carence du zinc dans les sols et les 
plantes, la baisse du rendement des paysans. Cette manière d'utilisation du DAP qui consiste à épandre l'engrais à la volée dans le champ n'est pas économique. Une des solutions idoines aux problèmes ci-dessus signalés serait la technique du micro dosage des engrais. La vulgarisation de cette technique a été lancée le 09 décembre 2009 au Mali. Depuis les paysans sont de plus en plus nombreux à y recourir dans leurs Champs : moins d'engrais chimique pour plus de rentabilité dans la production. Le micro dosage consiste, pour la même production, à utiliser $75 \mathrm{~kg}$ d'engrais par hectare pour une culture qui en demanderait $150 \mathrm{~kg}$. Il s'agit de creuser un poquet à côté du jeune plant et d'y placer trois (3 à 5) graines de DAP. Ainsi, la plante profite mieux de la fertilisation et le paysan fait une économie d'engrais (Saba et al., 2017). La production augmentera et la biodisponibilité du zinc moins perturbée, car la quantité de DAP en contact avec le zinc est moindre. Ainsi les céréales produites sur ces sols ne présenteront pas de carence en ce micronutriment pour le bonheur de la population.

\section{Conclusion}

$\mathrm{Au}$ cours de notre étude nous avons procédé dans un premier temps à la caractérisation physico-chimique du sol de PANA. Pour ce faire, différentes techniques ont été employées. De l'ensemble des résultats on retiendra que le sol de la zone est surtout constitué de quartz accompagné de la kaolinite. La capacité d'échange cationique 7,84 méq/100 g est faible. Nous avons aussi remarqué que le sol avec une teneur de 2,08\% en matière organique et un $\mathrm{pH}=4,9$ est moyennement organique et fortement acide. Dans une deuxième phase, nous avons étudié la rétention du zinc en fonction du DAP, du temps et du zinc appliqués. La capacité de rétention du zinc dépend de la quantité du DAP appliquée au sol et du temps. Le piégeage du zinc dépend aussi de la quantité du zinc appliqué. Nous pouvons donc conclure que cette rétention est gouvernée en grande partie par la réaction de précipitation entre les ions zinc (II) $\mathrm{Zn}^{2+}$ et les ions phosphate.

\section{CONFLIT D'INTERÊTS}

Les auteurs déclarent qu'il n'y a pas de conflit d'intérêts.

\section{CONTRIBUTION DES AUTEURS}

$\mathrm{AD}$ a initié ces travaux. $\mathrm{AD}, \mathrm{SD}, \mathrm{APD}$, ST ont réalisé les travaux d'échantillonnage sur le terrain. $\mathrm{AD}, \mathrm{BT}$ et $\mathrm{LE}$ ont effectué les manipulations pour la caractérisation physicochimiques des sols. AD et SD ont collecté, exploité les données et rédigé le manuscrit. MP, BBT et HK ont supervisé les travaux et la rédaction du manuscrit.

\section{REMERCIEMENTS}

Les auteurs remercient les techniciens des laboratoires de Sol-Eau-Plante du Centre de Recherche Agronomiques de l'Institut d'Economie Rurale de Sotuba, de physicochimique des matériaux de la Faculté des Sciences et Techniques et d'Arrhénius de l'Université de Stockholm. Ils remercient également ISP-UPPSALA(Suède) pour son appui financier et le Centre Régional de Recherche Agronomique de Sotuba (Mali) pour l'appui matériel et technique.

\section{REFERENCES}

AFNOR NF X31-107. 2003. Qualité du sol Détermination de la distribution granulométrique des particules du sol Méthode à la pipette. Thème: Propriétés physiques des sols.

Anonyme. 2013. Chimie du sol, $5^{\text {ème }}$ Version, Chapitre V, $3^{\text {ème }}$ Partie : Nutrition p 2-42.

Anonyme.2001. Projet. Les Aptitudes Agricoles et Pastorales des Sols dans les Pays du CILSS., p.164.

Adhikari T, Rattan RK. 2007. Distribution of Zinc Fractions in Some Major Soils of India and the Impact on Nutrition of Rice. Communications in Soil Science and 
Plant Analysis, 38(19): 2779-2798. DOI: 10.1080/00103620701663032.

Alloway BJ. 2008. Zinc in Soils and Crops nutrition ( $2^{\text {nd }}$ edition). Published by IZA and IFA: Brussels Belgium and Paris, France.135: 19-21.

Bationo A, Hartemink A, Lungu O, Naimi M, Okoth P, Smaling E, Thiombiano L. 2006. African Soils: their Productivity and Profitability of Fertilizer Use. Background paper presented for the African Fertilizer Summit 9-13 ${ }^{\text {th }}$ June 2006, Abuja, Nigeria, 26: 3-10.

Colinet G. 2003. Eléments trace métalliques dans les sols. Contribution à la connaissance des déterminants, et de leur distribution spatiale en région limoneuse. Thèse de Doctorat, Belge, Gembloux, Faculté Universitaire des Sciences Agronomiques, p.415.

Frossard E, Julien P, Neyroud JA, Sinaj S. 2004. Le phosphore dans les sols. Etats de la situation en Suisse. Cahier de l'environement $n^{\circ} 368$, Office fédéral de l'environnement, des forets et du paysage, Berne.180p.

Gertrud N. 2005. Zinc deficiency in soils and crops in the Niger inland delta in Mali. Memoires de DEA (the department of physical chemistry, inorganic chemistry and structural chemistry), Stockholm, Sweden-40p.

Handbook on Reference Methods for Soil Analysis.1992.

Maliki M, Ifijem IH. 2020. Growth Performance and Nutrient Availability of Tomatoes (Lycopersicum Esculentum) Seedlings in Anaerobie Soils. ChemSearch Journal, 11(1): 52-58 http://www.ajol.info/index.php/csj

Mulambuila Nkongolo M, Kamambo RM, Jadika CT, Tshibamba MMJ, Mukanya MKB. 2015. Etude comparative de quelques fertilisants (Bat-guano et DAP) sur le rendement du niébé (Vigna unguilata, L. Walp.) dans la région de
Gandajika (RDC). Journal of Applied Biosciences, $\quad$ 92(1). DOI : http://dx.doi.org/10.4314/jab.v92i1.9.

Michel MK, Joseph TU, Alain KM, Luciens NK. 2017. Réponse de huit variétés de haricot commun (Phaseolus vulgaris L.) à la fertilisation minérale dans la région de Kolwezi, Lualaba (RD Congo). Journal of Applied Biosciences, 111(1). DOI :

http://dx.doi.org/104314/jab.v111i1.6

Munroe J. 2018. Manuel sur la fertilité du sol ( $3^{\text {ème }}$ édition). Publication 611F. Ontario, Canada p.236.

Pardo MT, 2008. Influence of Phosphate on Zinc Reaction in Variable Charge Soils (Madrid, Spain). Communications in Soil Science and Plant Analysis, 30(5-6): 725737.

DOI:

10.1080/00103629909370241.

Pérez-Novo C, Bermúdez-Couso A, LópezPeriago E, Fernández-Calvino D, Arias-Estévez M, 2011. Zinc Adsorption in Acid Soils Influence of Phosphate (Ourense, Spain). Geoderma, 162: 358364.

DOI:

0.1016/j.geoderma.2011.03.008.

Pahlavan-Rad MR, Pessarakli M, 2009. Response of Wheat Plants to Zinc, Iron, and Manganese Applications and Uptake and Concentration of Zinc, Iron and Manganese in wheat Grains. Communications in Soil Science and Plant Analysis, 40(7-8): 1322-1332. DOI: 10.1080/00103620902761262.

Pichard A. 2005. Zinc et ses dérivés. INERIS Fiche de données Toxicologiques et Environnementales des Substances Chimiques (version n ${ }^{\circ}$ ). France ; 1-69.

Panfili F. 2004. Etude de l'évolution de la spéciation du zinc dans la phase solide d'un sédiment de curage contaminé, induit par phytostabilisation. Thèse de Doctorat. Ingénierie de l'Environnement. Université de Provence - Aix - Marseille I. France 227: 10 -12. 
Saba F, Taonda SJB, Serme I, Bandaogo AA, Sourwema AP, Kabré A, 2017. Effets de la Microdose sur la Production du Niébé, du Mil et du Sorgho en Fonction Toposéquence (Burkina Faso). International Journal of Biological and Chemical Sciences, 11(5): 2082-2092. DOI:

htps://dx.doi.org/10.4314/ijbcs.v11i5.12.

Theriant V, Kergna A, Traoré A, Teme B, Smale M, 2015. Revue de la Structure et de la Performance de la Filière Engrais au Mali pour l'USAID-Mali, 57 : 7-8.

Vega Flora A, Covelo Emma F, Vazquez Juan J, Luisa A, 2007. Influence of Mineral and Organic Components on Copper, Lead and Zinc Sorption by Acid Soils (Vigo, Spain). Journal of Environmental
Science and Health, part A, 42(14): 21672173. DOI: 10.1080/10934520701629682.

Van Der Pol F, Keita MK. 1998. Méthodes d'Analyses Sols - Eaux - Plantes. Cahier de Méthodes du Centre Régional de Recherche Agronomique de Sotuba de l'Institut d'Economie Rurale du Mali.

Xie C, Xu J, Tang J, Baig SA, Xu X. 2013. Comparison of Phosphorus Determination Methods by Ion Chromatography and Molybdenum Blue Methods. Communications in Soil Science and Plant Analysis. 44(17): 25352545.

DOI: 10.1080/00103624.2013.811518. 